\title{
A randomized, double-blind, active placebo-controlled study of efficacy, safety, and durability of repeated vs single subanesthetic ketamine for treatment-resistant depression
}

\author{
Paulo R. Shiroma ${ }^{1,2}$, Paul Thuras ${ }^{3}$, Joseph Wels ${ }^{4}$, C. Sophia Albott ${ }^{5}$, Christopher Erbes ${ }^{6}$, Susannah Tye ${ }^{7}$ and
} Kelvin O. Lim $^{8}$

\begin{abstract}
The strategy of repeated ketamine in open-label and saline-control studies of treatment-resistant depression suggested greater antidepressant response beyond a single ketamine. However, consensus guideline stated the lack of evidence to support frequent ketamine administration. We compared the efficacy and safety of single vs. six repeated ketamine using midazolam as active placebo. Subjects received either six ketamine or five midazolam followed by a single ketamine during 12 days followed by up to 6-month post-treatment period. The primary end point was the change from baseline in the Montgomery-Åsberg Depression Rating Scale (MADRS) score at $24 \mathrm{~h}$ after the last infusion. Fifty-four subjects completed all six infusions. For the primary outcome measure, there was no significant difference in change of MADRS scores between six ketamine group and single ketamine group at $24 \mathrm{~h}$ post-last infusion. Repeated ketamine showed greater antidepressant efficacy compared to midazolam after five infusions before receiving single ketamine infusion. Remission and response favored the six ketamine after infusion 4 and 5 , respectively, compared to midazolam before receiving single ketamine infusion. For those who responded, the median time-to-relapse was nominally but not statistically different ( 2 and 6 weeks for the single and six ketamine group, respectively). Repeated infusions were relatively well-tolerated. Repeated ketamine showed greater antidepressant efficacy to midazolam after five infusions but fell short of significance when compared to add-on single ketamine to midazolam at the end of 2 weeks. Increasing knowledge on the mechanism of ketamine should drive future studies on the optimal balance of dosing ketamine for maximum antidepressant efficacy with minimum exposure.
\end{abstract}

\section{Introduction}

Ketamine, a glutamate receptor-blocking drug approved by the U.S. Food and Drug Administration for anesthetic use, has become a target of research for its

\footnotetext{
Correspondence: Paulo R. Shiroma (paulo.shiroma@va.gov)

${ }^{1}$ Geriatric Psychiatrist, Minneapolis VA Health Care System, Mental Health Service Line, Minneapolis, MN, USA

${ }^{2}$ Assistant Professor, Department of Psychiatry, University of Minnesota, Minneapolis, MN, USA

Full list of author information is available at the end of the article Previous presentation: Presented in part at the Society of Biological Psychiatry 74th Annual Meeting, Chicago, Illinois, 16-18 May 2019.
}

antidepressant effects, and possible anti-suicidal effects. Single ketamine at subanesthetic dose of $0.5 \mathrm{mg} / \mathrm{kg}$ for $40 \mathrm{~min}$ has demonstrated improvement in mood within a few hours in some patients with treatment-resistant depression $(\mathrm{TRD})^{1,2}$. The peak antidepressant effect occurs at 24-h post-infusion with gradual loss of therapeutic benefit between 5 and 8 days ${ }^{1,3}$. The strategy of repeated ketamine $^{4-7}$ suggested that several infusions beyond a single ketamine increase antidepressant response and prolong its durability; however, open-label study designs or the use of saline as placebo limited more 
definite conclusions. We aimed to overcome these limitations by conducting a two-arm, randomized, active placebo-controlled trial. Through a total of six infusions in 12 days, we compared the efficacy and safety of six IV ketamine versus a single IV ketamine among patients with TRD. To balance the number of infusions, subjects in the single ketamine arm had five IV midazolam, a shortacting benzodiazepine and anesthetic agent, with fast onset of action, short elimination half-life, and similar time course of dissociative and nonspecific behavioral effects (e.g., sedation, disorientation) to ketamine ${ }^{8}$.

\section{Methods}

\section{Study design and patients}

The study was conducted at the Minneapolis Veterans Affairs Medical Center between April 2015 and March 2019. Subjects were outpatient, aged 18-75 years, met criteria for major depressive disorder (MDD) by the Structured Clinical Interview for DSM-IV(SCID), and had lack of response to at least two adequate antidepressant trials of different pharmacological classes during current major depressive episode (MDE) according to the Antidepressant Treatment History Form (ATHF) ${ }^{9}$. Systematic evaluation of previous antidepressants was assessed on all available information including VA pharmacy, and community-based records. In addition, participants had a score $\geq 32$ on the Inventory of Depressive Symptomatology-Clinician Rated (IDS-C30) ${ }^{10}$ for severity of MDE at screening. Current antidepressant dosages including augmenting agents and/or frequency and duration of psychotherapy sessions remained stable for $\geq 6$ weeks prior to consent and throughout the study.

Patient were excluded if they had a lifetime DSM-IV criteria for post-traumatic stress disorder (PTSD), mild to moderate traumatic brain injury, psychosis-related disorder, bipolar disorder, or any Axis I disorder other than MDD as the primary presenting problem. Patients with a history of alcohol or substance use disorder within 6 months of screening, imminent risk of suicidal/homicidal ideation and/or behavior with intent, a Mini-Mental State Examination score $\leq 27$, positive urine toxicology or pregnancy test were also excluded. Medical records and laboratory tests were reviewed for any unstable medical illness. Study anesthesiologist were consulted on those cases with possible medical contraindication to participate.

\section{Study procedures}

Randomization was conducted using permutated blocks of 4 and 1:1 assignment between treatments. The patients received six infusions on a Monday-Wednesday-Friday schedule over a 12-day period. Patients received five midazolam infusions followed by a single ketamine or six ketamine treatments (see Fig. SF1). The fact that the last infusion consisted of ketamine for both arms was kept undisclosed to subjects and raters of antidepressant outcomes at $24 \mathrm{~h}$. Each infusion of ketamine at $0.5 \mathrm{mg} / \mathrm{kg}$ or midazolam at $0.045 \mathrm{mg} / \mathrm{kg}$ lasted $40 \mathrm{~min}$. The selection of ketamine and midazolam doses was based on previous $\mathrm{RCTs}^{1,11}$. The dose of ketamine and midazolam were calculated by ideal body weight based on sex, age, height, and body frame in the Metropolitan Life Insurance tables. Group assignments for each participant was concealed in sequentially numbered, sealed, opaque envelopes with drug identity by the research pharmacist; investigators, raters, and patients were masked to treatment assignment.

Patients arrived at the infusion unit after fasting for at least $8 \mathrm{~h}$. An indwelling catheter was placed in peripheral vein of non-dominant arm for medication administration; monitor of pulse, blood pressure, digital pulse oximetry, and respiratory rate was recorded every $10 \mathrm{~min}$ for $1 \mathrm{~h}$ beginning $10 \mathrm{~min}$ before infusion. Trained raters administered pre-infusion rating scales and repeated them again during a 2 -h post-infusion monitoring period. A study physician was present throughout the infusion and study anesthesiologist (J.W.) was reached, if necessary. Medications such as labetalol, hydralazine, ondansetron, and flumenazil as well as a crash cart were available to manage adverse side effects. Guidelines established for clinically significant changes in vital signs and mental status during the ketamine infusions were as follows: systolic blood pressure $>161$ or $<89$, diastolic BP $>110$ or $<40$; heart rate $<40$ or $>130$ beats/min; respiratory rate $<10$ or $>30$ per minute; pulse oximetry $<90 \%$; severe hallucinations, confusion, delusions, irrational behavior, or agitation. Before leaving the infusion unit, subjects demonstrated that all clinically significant side effects were resolved by a score $\geq 9$ in the modified Aldrete scoring system ${ }^{12}$. Written instructions about potential side effects of sedatives and several measures to improve recovery at home (e.g., proper hydration and rest; abstain from alcohol) were provided at discharge to patient and companion adult.

\section{Outcomes}

The study assessments were performed at days $0,1,3,5$, 8,10 , and 12 to assess the safety and efficacy of ketamine compared to active placebo during infusion phase. Posttreatment assessments were measured at weekly intervals for the first 4 weeks, at 2-week intervals for the next 8 weeks, and at 4-week intervals for the remaining 12 weeks. The primary outcome was change in depression severity measured by the clinician-administered Montgomery-Åsberg Depression Rating Scale score $(\mathrm{MADRS})^{13}$ at $24 \mathrm{~h}\left(\mathrm{~T}_{+24}\right)$ after the last infusion. Raters were trained in MADRS prior to and during study with a level of interrater reliability of 0.92 . Secondary outcomes included change in MADRS score over time, rates of 
response ( $\geq 50 \%$ MADRS reduction in the baseline score), rates of remission (MADRS $<10$ ), rates of response and remission over time, and durability of response for up to 6 months. Other secondary outcomes included clinical global impression (CGI) severity and improvement measures $^{14}$, self-reported numeric rating scale (NRS) for pain $^{15}$, Beck anxiety inventory (BAI) ${ }^{16}$, and credibility and expectancy questionnaire (CEQ) ${ }^{17}$ for clinical treatment. Cognitive performance (CogState $)^{18}$, and functional neuroimaging on a sub-sample of patients will be reported separately.

General side effects were measured by the patient rated inventory of side effects (PRISE). Psychotogenic effects were measured with the four-item positive symptom subscale of the Brief Psychiatric Rating Scale $(\text { BPRS }+)^{19}$ consisting of suspiciousness, hallucinations, unusual thought content, and conceptual disorganization; dissociative effects and manic symptoms were measured with the Clinician-Administered Dissociative States Scale $(\mathrm{CADSS})^{20}$ and Young Mania Rating Scale (YMRS) ${ }^{21}$, respectively. Additionally, The Columbia-Suicide Severity Rating Scale (C-SSRS) Screening Version-Since Last $\mathrm{Visit}^{22}$ was rated during and after infusion phase for safety purposes.

\section{Statistical analysis}

The power analysis was performed for MADRS score under the following assumptions: (1) analyses of covariance with the main effects of treatment (a single versus six infusions) and time (0 and Day 13), and the treatment by time interaction; (2) compound symmetric covariance matrix, and (3) 5\% significance level. Our previous openlabel study of six repeated ketamine in TRD patients ${ }^{4}$ showed a difference of 23 points in MADRS score from baseline to $24 \mathrm{~h}$ after the last infusion (mean MADRS score $=29.9, \mathrm{SD}=2.3$ to mean MADRS score $=7.0, \mathrm{SD}$ $=2.3$ ). Based on these results, we estimated that a sample size of 21 patients per group was powered to detect a 10point difference in change of MADRS score between the single versus repeated ketamine group. Repeated measures ANOVA was used to test for differences between groups in change from baseline to $\mathrm{T}_{+24}$ after the last infusion and baseline and $\mathrm{T}_{+24}$ after the fifth infusion. Multi-level models were used to compare groups on change across all MADRS measures. All tests were twosided with significance at $p<0.05$.

\section{Ethics approval}

The authors assert that all procedures contributing to this work comply with the ethical standards of the relevant national and institutional committees on human experimentation and with the Helsinki Declaration of 1975, as revised in 2008. All procedures involving human subjects/patients were approved by Minneapolis VA
Health Care System Institutional Review Board (protocol number 4533-B).

\section{Results \\ Study participants}

One-hundred seventy-eight individuals were prescreened for eligibility through consultation with study physicians. Of these, 62 signed consent forms and underwent formal in-person screening (see the CONSORT diagram in Fig. SF2). There were four participants who failed screening; 58 subjects were randomized to treatment with four patients dropping prior to baseline data collection. All 54 subjects with baseline assessment, initiated study interventions and completed primary outcome end point. Five midazolam plus single ketamine group was composed by 29 subjects; a total of 25 subjects were in the six-ketamine group.

Participants' baseline characteristics are summarized in Table 1. There was no significant difference between groups for demographic and clinical characteristics. The sample was mostly composed by middle aged, unemployed or retired, married, white males with a history of mood disorder among first-degree relatives. Patients had also a chronic history of depression that included at least one past psychiatric hospitalization with almost half of the patients reporting a previous suicidal attempt. Overall, patients had a current MDE with severe symptoms that failed to more than 02 adequate antidepressant trials and augmenting agents.

\section{Primary outcome}

There was no significant difference between single (MADRS mean change $=21.0,95 \% \mathrm{CI}=17.2-24.8)$ versus six ketamine treatments (MADRS mean change $=$ $17.2,95 \% \mathrm{CI}=13.2-21.2)$ at $\mathrm{T}_{+24}$ after the end of treatment (six infusions) $\left(F_{1,52}=2.41, P=0.13, \eta \mathrm{p} 2=0.044\right)$ (Fig. 1). There was a significant change in MADRS score by groups over time when including all MADRS measures $\left(F_{11,541.11}=2.63, P=0.003\right)$. The mean MADRS score among subjects receiving ketamine was significantly lower by 8.07 ( $95 \% \mathrm{CI}, 1.67-14.46)$ prior to infusion $5\left(F_{1,95.7}=\right.$ 6.28, $P=0.014)$, by $8.29(95 \% \mathrm{CI}, 1.87-14.70)$ at $\mathrm{T}_{+24}$ after infusion $5\left(F_{1,96.7}=6.58, P=0.012\right)$ and by $6.40(95 \%$ CI, 0.01-12.79) prior to infusion $6\left(F_{1,95.7}=3.94, P=\right.$ $0.050)$ compared to subjects receiving midazolam.

\section{Secondary outcomes \\ Response and remission rates}

The response rates were not significantly different between groups at the end of six infusions $\left(X^{2}{ }_{1 \mathrm{df}}=0.73\right.$, $P=0.39$ ) (Fig. 2a). There was also no significant difference in remission rates at $\mathrm{T}_{+24}$ after the last infusion between repeated midazolam plus single ketamine versus repeated ketamine $\left(X_{1 \mathrm{df}}^{2}=0.56, P=0.46\right)($ Fig. $2 \mathrm{~b})$. Throughout the 
infusions phase, there was a significant difference in remission rates at $\mathrm{T}_{+24}$ after infusion 4 (six ketamine $=$ $54.2 \%$ versus midazolam $=17.9 \% ; X^{2}{ }_{1 \mathrm{df}}=7.53, P=0.006$ ) and in response rates at $\mathrm{T}_{+24}$ after infusion 5 (six ketamine $=76 \%$ versus midazolam $=39.3 \% ; X^{2}{ }_{1 \mathrm{df}}=7.25, P=$ 0.007).

\section{Other secondary outcomes}

There was non-significant difference in anxiety as measured by BAI score change over times between groups at the end of infusion $5\left(F_{4,182.28}=1.74, P=0.14\right)$ and infusion $6\left(F_{5,234.50}=1.53, P=0.18\right)$. Self-rated pain also did not show significant difference between groups over infusion time points $\left(F_{5,238.34}=1.29, P=0.27\right)$. There was a significant improvement in CGI for subjects in both groups, midazolam plus single ketamine (baseline mean $=$ $5.14,95 \% \mathrm{CI}=4.75-5.53$ to post-infusion mean $=2.63$, 95\% $\mathrm{CI}=2.14-3.12) \quad(t(56)=8.01, p<0.001)$ and six ketamine (baseline mean $=5.32,95 \% \mathrm{CI}=4.90-5.74$ to post-infusion mean $=2.01,95 \% \mathrm{CI}=1.55-2.48)(t(48)=$ 10.63, $p<0.001)$ without significant difference between groups $\left(F_{1,54.83}=3.59, P=0.06\right)$ at $\mathrm{T}_{+24}$ after the end of treatment. There was no significant difference between groups in credibility $\left(F_{1,52}=0.19, P=0.66\right)$ and expectancy $\left(F_{1,52}=0.03, P=0.86\right)$ of treatment as measured by CEQ scores. There was no significant difference in the mean change of MADRS score from baseline to $T_{+24}$ after the last infusion between subjects taking benzodiazepines $(N=11$, mean change MADRS score $=22.9, \mathrm{SD}=9.5)$ or not taking benzodiazepines $(N=13$, mean change in MADRS score $=23.2, \mathrm{SD}=6.5)\left(\mathrm{F}_{1,22}=0.01, P=0.94\right)$ in the six ketamine group.

\section{Durability of response}

MADRS scores at weekly for 4 weeks, biweekly for 8 weeks, and monthly for 3 months were used to determine time-to-relapse (MADRS $>50 \%$ from baseline) among patients who achieved response after the last infusion $(N=20$ for midazolam plus single ketamine and $N=19$ for six ketamine). The Kaplan-Meier estimates of the 6-month relapse rates after response (Fig. 3) was 75\% $(95 \% \mathrm{CI}=54.2-95.8 \%)$ and $68.4 \%(95 \% \mathrm{CI}=45.4-91.4 \%)$ for midazolam plus single ketamine and six ketamine group, respectively. The long-rank test revealed a nonstatistically significant difference between relapse rates over time $\left(X^{2}{ }_{1 \mathrm{df}}=1.61 ; P=0.21\right)$. The median time-torelapse was 2 weeks for the midazolam plus single ketamine group, and 6 weeks for the six-ketamine group (95\% $\mathrm{CI}$ for the hazard ratio $=0.3$ to $1.4 ; P=0.27$ ).

\section{Adverse events}

During infusion phase, the most common side effects for ketamine were general malaise (28.0\%), decreased energy $(28.0 \%)$, increased in blood pressure $(24.0 \%)$,
Table 1 Baseline demographic and clinical characteristics of patients with treatment-resistant depression treated with five midazolam plus a single ketamine versus six ketamine infusions.

\begin{tabular}{|c|c|c|c|c|c|c|}
\hline \multirow[t]{3}{*}{ Characteristics } & \multirow{2}{*}{\multicolumn{2}{|c|}{$\begin{array}{l}\text { Six } \\
\text { ketamine }\end{array}$}} & \multirow{2}{*}{\multicolumn{2}{|c|}{$\begin{array}{l}\begin{array}{l}\text { Five } \\
\text { midazolam } \\
\text { plus single } \\
\text { ketamine }\end{array} \\
(N=29)\end{array}$}} & \multirow{2}{*}{\multicolumn{2}{|c|}{$\begin{array}{l}\text { Overall sample } \\
(N=54)\end{array}$}} \\
\hline & & & & & & \\
\hline & Mean & SD & Mean & SD & Mean & SD \\
\hline Age (years) & 54.4 & 13.8 & 51.2 & 12.5 & 52.7 & 13.1 \\
\hline Level of education (years) & 14.5 & 2.1 & 14.7 & 2.0 & 14.6 & 2.0 \\
\hline Age of first MDE (years) & 25.6 & 11.6 & 25.0 & 10.1 & 25.3 & 10.4 \\
\hline Number of lifetime MDE & 5.3 & 2.6 & 6.1 & 3.8 & 5.7 & 3.3 \\
\hline Duration of current MDE (weeks) & 78.3 & 39.6 & 84.9 & 35.3 & 81.9 & 37.1 \\
\hline $\begin{array}{l}\text { Number of adequate antidepressant } \\
\text { trials during current MDE }\end{array}$ & 2.2 & 0.4 & 2.4 & 1.1 & 2.3 & 0.8 \\
\hline $\begin{array}{l}\text { Lifetime number of } \\
\text { antidepressant trials }\end{array}$ & 4.6 & 1.9 & 4.5 & 2.0 & 4.5 & 1.9 \\
\hline \multirow[t]{2}{*}{ IDS-C } & 36.8 & 3.9 & 39.8 & 7.3 & 38.4 & 6.1 \\
\hline & $\mathrm{N}$ & $\%$ & $\mathrm{~N}$ & $\%$ & $\mathrm{~N}$ & $\%$ \\
\hline Male & 22 & 88.0 & 24 & 82.8 & 46 & 85.2 \\
\hline \multicolumn{7}{|l|}{ Race } \\
\hline White & 21 & 84.0 & 25 & 86.2 & 46 & 85.2 \\
\hline Black/African American & 3 & 12.0 & 1 & 3.4 & 4 & 7.4 \\
\hline Other & 1 & 4.0 & 3 & 10.3 & 4 & 7.4 \\
\hline Married & 17 & 68.0 & 12 & 41.4 & 29 & 53.7 \\
\hline Unemployed/retired & 16 & 64.0 & 19 & 65.5 & 35 & 64.8 \\
\hline Melancholic depression & 8 & 32.0 & 6 & 20.7 & 14 & 25.9 \\
\hline \multicolumn{7}{|l|}{ Comorbid conditions } \\
\hline Anxiety spectrum & 4 & 17.4 & 5 & 20.0 & 9 & 16.7 \\
\hline Dysthymia & 6 & 24.0 & 5 & 17.2 & 11 & 20.4 \\
\hline Sub-syndromal PTSD & 6 & 24.0 & 5 & 17.2 & 11 & 20.4 \\
\hline Personality disorder & 4 & 16.0 & 2 & 6.9 & 6 & 11.1 \\
\hline Chronic pain & 10 & 40.0 & 9 & 31.0 & 19 & 35.2 \\
\hline Sleep disorder & 14 & 56.0 & 14 & 48.3 & 28 & 51.9 \\
\hline Tobacco disorder & 22 & 88.0 & 23 & 79.3 & 51 & 94.4 \\
\hline Severe MDE & 15 & 60.0 & 11 & 37.9 & 26 & 48.1 \\
\hline History of AUD/SUD & 10 & 40.0 & 8 & 27.6 & 18 & 33.3 \\
\hline \multicolumn{7}{|l|}{ Family history of } \\
\hline Mood disorder & 19 & 76.0 & 18 & 62.1 & 37 & 68.6 \\
\hline Other psychiatric disorder & 3 & 12.0 & 7 & 24.1 & 10 & 18.5 \\
\hline AUD/SUD & 9 & 36.0 & 11 & 37.9 & 20 & 37.0 \\
\hline Previous suicidal attempt & 12 & 48.0 & 14 & 48.3 & 26 & 48.1 \\
\hline Previous psychiatric hospitalization & 17 & 58.6 & 18 & 72.0 & 35 & 64.8 \\
\hline Past ECT treatment & 4 & 16.0 & 7 & 24.1 & 11 & 20.4 \\
\hline \multicolumn{7}{|l|}{ Concurrent use of } \\
\hline Augmenting agents & 18 & 72.0 & 21 & 72.4 & 39 & 72.2 \\
\hline Benzodiazepines & 12 & 48.0 & 11 & 37.9 & 23 & 42.6 \\
\hline Psychotherapy & 9 & 36.0 & 12 & 41.4 & 21 & 38.9 \\
\hline
\end{tabular}

headaches (24.0\%), fatigue (24.0\%), nausea/vomiting (20.0\%), anxiety (20.0\%), and poor concentration (20.0\%). Within the same period, the most common adverse events for midazolam plus single ketamine were anxiety (24.1\%), decreased energy $(17.2 \%)$, increased in blood pressure (17.2\%), fatigue (13.8\%), and headaches (13.8\%). 


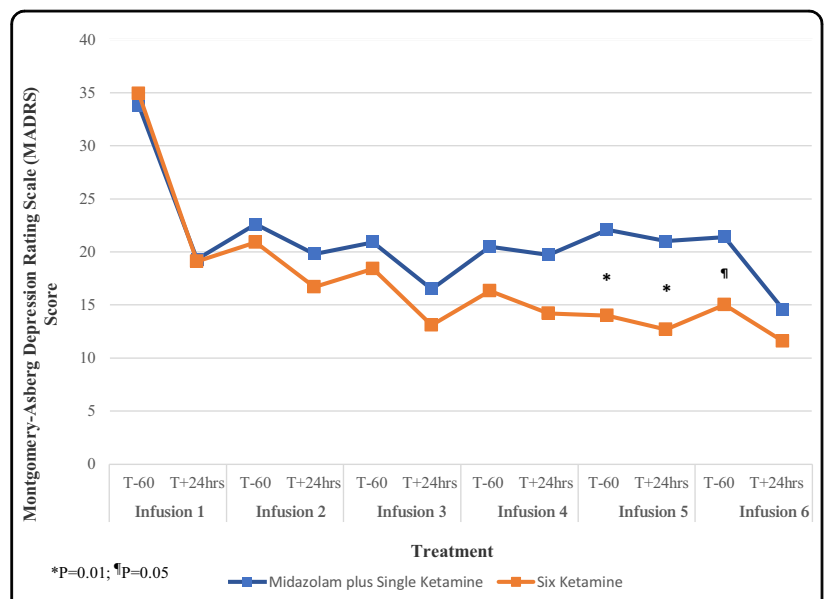

Fig. 1 Mean Change in Severity of Depression between Six Ketamine versus Midazolam plus Single Ketamine Treatment within a 12-day Period. Severity of depression scores measured by the Montgomery-Åsberg Depression Rating Scale (MADRS) over 12 days between six ketamine and five midazolam plus single ketamine (last infusion) treatment groups in a randomized controlled trial in treatment-resistant major depression. Figure depicts mean MADRS scores in patients with treatment-resistant depression before and after $24 \mathrm{~h}$ of each ketamine $(0.5 \mathrm{mg} / \mathrm{kg})$ or single ketamine $(0.5 \mathrm{mg} / \mathrm{kg})$ preceded by five midazolam $(0.045 \mathrm{mg} / \mathrm{kg})$ infusion. * indicates significant difference in MADRS score between treatment condition $(p=0.01)$ before and at $24 \mathrm{~h}$ after infusion 5. "Indicates significant difference in MADRS score between treatment conditions $(p=0.05)$ before infusion 6 .

\section{Vital signs}

There were 25 and 17 cases of mild and moderate adverse events during infusion phase, respectively. Moderate side effects were related to transient hypertensive episode (systolic $>161$ or diastolic $>110$ per protocol) that require at least one dose of antihypertensive medication such as labetalol or hydralazine as recommended by study anesthesiologist. Moderate adverse events also included nausea that required IV medication (e.g., ondansetron). Mild cases included increased blood pressure or nausea that subsided without medications, and IV site bruising but not pain. During infusions $1-5$, there was a significantly higher systolic and diastolic blood pressure at $\mathrm{T}_{+40 \mathrm{~m}}$ (systolic mean difference $=25.6, \quad 95 \%$ CI: 16.4-34.9; diastolic mean difference $=12.9,95 \% \mathrm{CI}$ : 7.2-18.8) and diastolic blood pressure at $\mathrm{T}_{+100 \mathrm{~m}}$ (mean difference $=12.9,95 \% \mathrm{CI}: 3.6-22.2$ ) for the ketamine group compared to midazolam. There was no significant difference in heart rate between groups at any time point during infusions (data not shown).

\section{Dissociative, psychomimetic, and mania}

During infusions $1-5$, there was a significantly greater dissociation measured by CADSS score at $\mathrm{T}_{+40 \mathrm{~m}}$ (mean difference $=12.8,95 \% \mathrm{CI}: 10.1-15.6)$ for the ketamine group $\quad($ mean $=15.3 \quad 95 \%$ CI: 13.3$) \quad$ compared to

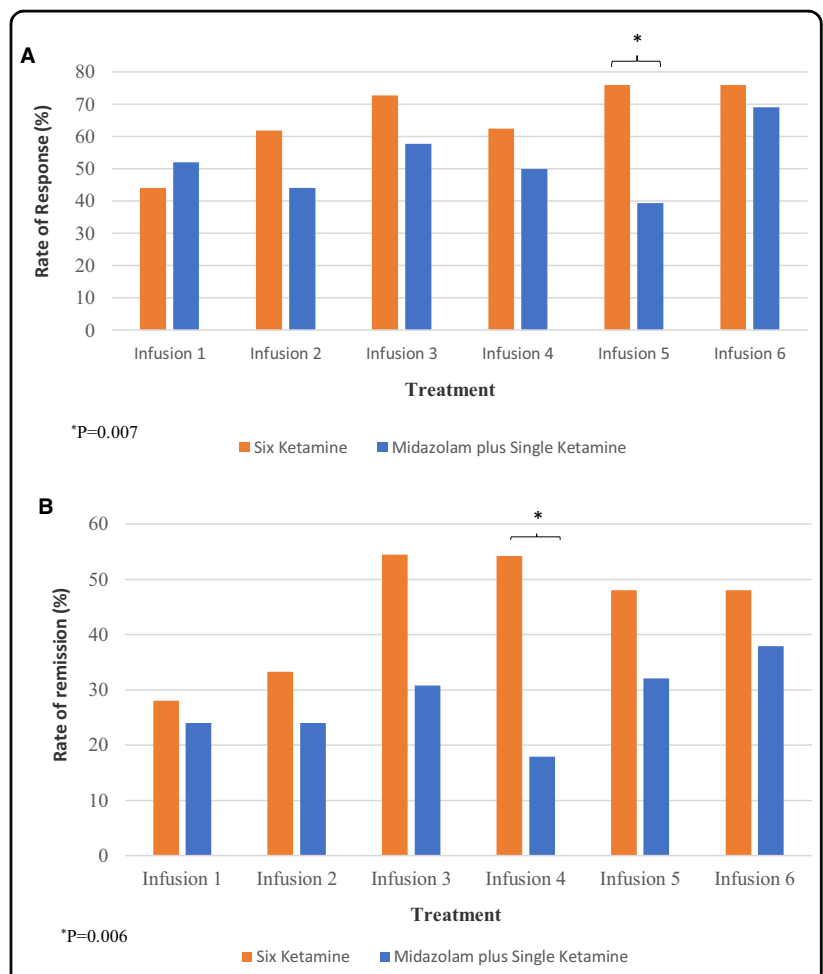

Fig. 2 Antidepressant Response and Remission Rates between Six Ketamine versus Midazolam plus Single Ketamine Treatment within a 12-day Period. a Response rates over 12 days between six ketamine and five midazolam plus single ketamine (last infusion) treatment groups in a randomized controlled trial among subjects with treatment-resistant major depression. Response is defined as > 50\% decrease from baseline score measured by the MontgomeryÅsberg Depression Rating Scale. Figure depicts response in patients with treatment-resistant depression measured at $24 \mathrm{~h}$ after each ketamine $(0.5 \mathrm{mg} / \mathrm{kg})$ or single ketamine $(0.5 \mathrm{mg} / \mathrm{kg})$ preceded by five midazolam $(0.045 \mathrm{mg} / \mathrm{kg})$ infusion. * indicates significant difference in response between treatment conditions $(p=0.007)$ at $24 \mathrm{~h}$ after infusion 5 (Fig. 2b). Remission rates over 12 days between six ketamine and five midazolam plus single ketamine (last infusion) treatment groups in a randomized controlled trial among subjects with treatment-resistant major depression. Remission is defined as score < 10 in the Montgomery-Åsberg Depression Rating Scale. Figure depicts remission in patients with treatment-resistant depression measured at $24 \mathrm{~h}$ after each ketamine $(0.5 \mathrm{mg} / \mathrm{kg})$ or single ketamine $(0.5 \mathrm{mg} / \mathrm{kg})$ preceded by five midazolam $(0.045 \mathrm{mg} / \mathrm{kg})$ infusion. ${ }^{*}$ indicates significant difference in remission between treatment conditions ( $p=$ $0.006)$ at $24 \mathrm{~h}$ after infusion 4.

midazolam (mean $=2.5$ 95\% CI: 0.6-4.4) (see Fig. SF3). All patients receiving ketamine had complete resolution of dissociative side effects within the 2-h monitoring period. Participants' dissociative side effects (change in CADSS score a $T_{+40 \mathrm{~m}}$ ) were non-significantly correlated with antidepressant response (change in MADRS score) at $\mathrm{T}_{+24 \mathrm{~h}}$ post-infusion for midazolam (Pearson's $r=-0.03$, $p=0.89$ ) or ketamine (Pearson's $r=0.04, p=0.86$ ). Comparison of change in CADSS scores in the ketamine group at $\mathrm{T}_{+40 \mathrm{~m}}$ (peak of side effects) revealed non- 


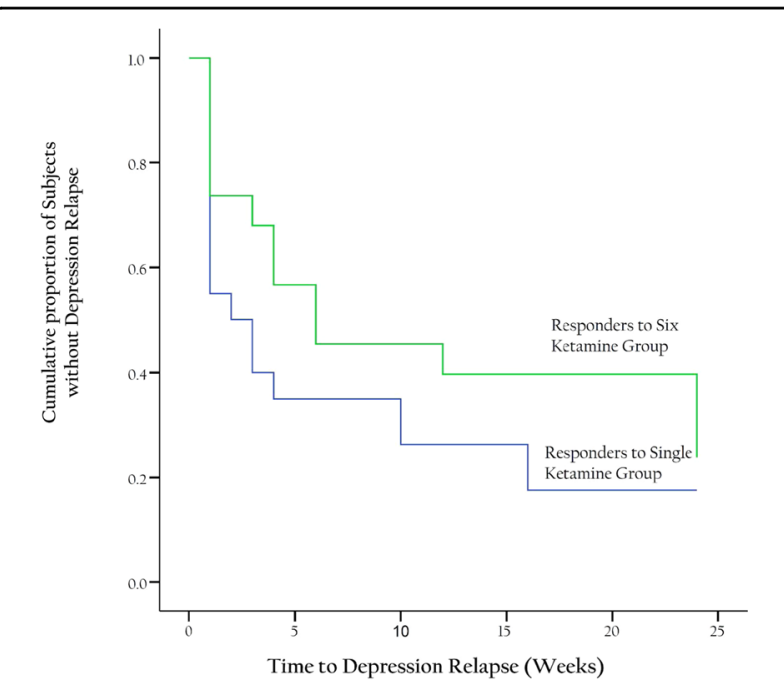

Fig. 3 Time-to-relapse between responders to single vs six ketamine infusions in treatment-resistant depression. Figure depicts Kaplan-Meier analysis of responders for up 6 months of follow-up. Relapse was defined as $<50 \%$ improvement in

Montgomery-Åsberg Depression Rating Scale score at that visit compared with baseline.

significant change in dissociative side effects with repeated infusions $\left(F_{5,220.28}=1.83, P=0.11\right)$. There was not significant change within or between groups regarding psychotic (BPRS+) (see Fig. SF4) or elevated mood symptoms (YMRS-item 1) at any time point throughout infusions.

\section{Serious adverse events}

There were two cases deemed as a serious adverse event that required IRB report (see Table ST1): one patient (assigned to midazolam) complained of headaches triggered by loud noises and bright lights after post-infusion MRI as part of study protocol; another patient (assigned to ketamine) reported mild headaches during second infusion, which increased during follow-up phase. Headaches in both cases were considered unrelated to study drugs and eventually subsided. Through interviews with subjects, no suicidal attempts nor drug-seeking behavior were elicited throughout the study.

\section{Blinding}

Subjects were asked prior to and at the end of the last infusions $\left(\mathrm{T}_{+160}\right)$ about treatment allocation. Prior to the last infusion (midazolam versus ketamine), 6.3\% among midazolam cases and $56.3 \%$ among ketamine cases were erroneous about what treatment they were assigned to $\left(X_{1 \mathrm{df}}^{2}=9.30 ; P=0.002\right)$. At the end of the last infusion (midazolam plus single ketamine versus six ketamine), $10.7 \%$ among midazolam cases and 50.0\% among ketamine cases guessed incorrectly about assigned treatment
$\left(X_{1 \mathrm{df}}^{2}=9.12 ; P=0.002\right)$. Raters, who were different during infusions days from those rating antidepressant outcomes at $24 \mathrm{~h}$., incorrectly guessed $20.8 \%$ of midazolam cases and $7.1 \%$ of repeated ketamine cases prior to the last infusion $\left(X^{2}{ }_{1 \mathrm{df}}=1.25 ; P=0.26\right)$. After the last infusion, 6.9 and $26 \%$ raters were incorrect about treatment assignment among midazolam plus single ketamine and repeated ketamine cases, respectively $\left(X^{2}{ }_{1 \mathrm{df}}=3.62 ; P=\right.$ 0.06).

\section{Discussion}

This single center study of Veterans with moderate-tosevere, recurrent, TRD showed that for the primary outcome measure, there was no significant difference in change of MADRS scores between groups at $24 \mathrm{~h}$ postlast infusion at the end of 12 days of treatment. Repeated ketamine showed greater antidepressant efficacy compared to midazolam after five infusions before receiving single ketamine as the last infusion. The rate of remission and response was greater among subjects in the sixketamine group after infusion 4 and 5, respectively. For those subjects who responded to either assigned treatment, six ketamine tripled the median time-to-relapse over a 6-month period as compared to those in the midazolam plus single ketamine group; however, it fell short of statistically significant difference. Transient psychoactive and hemodynamic effects during ketamine infusion were consistent with those in previous reports ${ }^{7,6}$ and did not increase when repeated treatments were given in a short-term fashion.

The prospect of whether repeated dosing of ketamine offer safe and superior antidepressant outcomes is a critical question as studies have shown that frequent use of ketamine could lead to cognitive impairments ${ }^{23}$, dissociation, and poor impulse control ${ }^{24}$. Medical, legal, and even ethical concerns of using repeated ketamine to treat psychiatric conditions have been raised ${ }^{25}$. Despite the small sample in this study, this is the largest randomized study that compare repeated ketamine versus an active placebo in TRD. Previously, Singh and colleagues ${ }^{26}$ showed that both twice-weekly and thrice-weekly administration of ketamine over 15 days of treatment had greater antidepressant efficacy versus saline (leastsquare mean change in MADRS score of 16.0 and 16.4 points, respectively). An open-label study of thrice ketamine weekly for 2 weeks among unipolar and bipolar depressed Chinese patients ${ }^{7}$ showed an overall reduction of 15.5 and 18.7 points in MADRS score at $24 \mathrm{~h}$. after ketamine infusion 5 and 6, respectively. A more recently study among patients with TRD who responded and then relapsed after a cross-over single ketamine (versus midazolam $)^{6}$ showed a reduction of 12 points in MADRS score after completing an open-label treatment of thrice a week for 2 weeks. 
From these previous studies, our findings support the idea that repeated ketamine further reduce the severity of depression in TRD. However, the antidepressant improvement through six ketamine treatments was not significantly different when compared to a single ketamine. Previous reports on repeated ketamine consistently found that the largest improvement $(\geq 50 \%)$ in TRD occurred after the first infusion ${ }^{26,7,6,4,27,28}$. It is likely then that the relatively small sample in our study was insufficient to capture any significant difference between antidepressant gain beyond the first infusion in the repeated ketamine group versus that in the single ketamine. On the other hand, subjects in the repeated ketamine group achieved a mean group treatment difference of -4.0 against repeated midazolam plus single ketamine at $24 \mathrm{~h}$ after the last infusion. A 4-point difference on the MADRS has been suggested as a stringent criterion for judging whether an effect size is clinically relevant ${ }^{29}$ with more recent reports suggesting a minimal clinically important difference threshold of 2 points in MADRS ${ }^{30}$. Moreover, Popova and colleagues ${ }^{31}$ on the recent approval of esketamine, the S-enantiomer of ketamine racemate, by the U.S. Food and Drug Administration for TRD in adults, reported a significant difference of four points at day 28 in the change of MADRS in a multicenter phase 3 RCT comparing esketamine plus antidepressant versus antidepressant plus placebo. Thus, we argue that despite the type II error in our study, there was a clinically significant difference supporting the use of repeated infusions.

Our study also support recent review of masking in ketamine studies for mood disorders ${ }^{32}$ namely that midazolam as a comparator yields smaller effects of ketamine than those which used saline. However, participants at the dose of midazolam used in this study $(0.045 \mathrm{mg} / \mathrm{kg})$ had lower intensity of dissociative symptoms as compared to ketamine, and in fact, correctly guessed treatment assignment in more than $90 \%$ of the cases even before exposure to ketamine at the last infusion. Therefore, while the use of an active placebo in repeated ketamine administration is encouraged to avoid overestimating ketamine's antidepressant effect, an optimal comparator that mimic the dissociative and psychomimetic side effects of ketamine and preserve masking is still missing. As an alternative, low dose of ketamine such as $0.2 \mathrm{mg}$ / $\mathrm{kg}^{11,33}$ have shown to lack antidepressant effect while still inducing dissociative effects.

In general, serial ketamine had greater side effects as compared to midazolam plus single ketamine. Acute, mild, and transient dissociation was the most common side effect reported on ketamine dosing days with other treatment emergent adverse events appeared similar in frequency as reported previously (e.g., increased in blood pressure, decreased energy, headache, nausea, etc.).
The frequency of dissociative side effects did not appear to change with subsequent ketamine infusions and was not associated with antidepressant response. Although there have been reports of an association between dissociative side effects and antidepressant response to ketamine ${ }^{34,35}$, additional research is required to further explain whether dissociation account for antidepressant improvement.

We found that during the 6-month follow-up among those who responded to either assigned treatment after six infusions, multiple ketamine prolonged the response for a median of 6 weeks compared to 2 weeks of midazolam plus single ketamine. Considering that response to a single dose of ketamine tends to dissipate typically 2 weeks if ketamine was not repeated, an effective strategy to maintain response after cessation of infusions is critical. Phillips and colleagues ${ }^{6}$ demonstrated that four weekly administration of intravenous ketamine maintain response after an acute open-label ketamine treatment of thrice a week for 2 weeks. Similarly, patients who achieved stable remission and stable response after 16 weeks of initial treatment with esketamine, decreased the risk of relapse by $51 \%$ and $70 \%$, respectively, during a maintenance phase of esketamine and antidepressant treatment compared to antidepressant and placebo treatment ${ }^{36}$. These findings support the possibility that repeated ketamine in combination to conventional antidepressants is a feasible strategy to maintain treatment response. Safety data and the optimal benefit-risk ratio for long-term treatment with ketamine is warranted.

The immediate and delayed antidepressant effects of ketamine, which are independent of its sustained blood concentrations, could be based on different mechanisms. Studies have focused on the non-competitive antagonist action on the NMDA receptor and subsequent activation of AMPA receptors driving acute antidepressant effect $^{37,38}$. The modulation of glutamate receptors triggers downstream the modulation of synthesis and release of brain-derived neurotrophic factor and enhances synaptic plasticity via activation of molecular targets such as mammalian target of rapamycin and eukaryotic elongation factor 2. These systems may be involved in the delayed maintenance of response rather than the acute and rapid antidepressant effect of ketamine. Recent preclinical studies support this idea by showing that ketamine's effects on behavior and ensemble activity occur rapidly and precede its effects on spine formation in the prefrontal cortex ${ }^{39}$. These newly formed spines are not required for inducing ketamine's effects acutely but are critical for sustaining the antidepressant effect over time. More recently, preliminary clinical findings suggest a central role for opiate agonism in the antidepressant effects of ketamine ${ }^{40}$ although conflicting results were also reported ${ }^{41}$. Overall, a growing body of evidence indicates that additional mechanisms, not mutually 
exclusive and possibly complementing each other, are likely mediating the unique properties of ketamine and its ketamine metabolites [e.g., (2R,6R)-hydroxynorketamine] as antidepressant ${ }^{42}$.

\section{Limitations of the study}

The study for the primary outcome was underpowered despite surpassing recruitment goal for a total of 54 participants. A total of 87 patients per group would have needed to detect the difference of 4 points in MADRS score between groups at end of six infusions (21 versus 17.2). The study design aimed to maximize recruitment while responding a pragmatic question on the efficacy of repeated ketamine. Ideally, a trial would have included an additional placebo-controlled arm composed exclusively by midazolam infusions. However, such a design would have been needed much more expensive, more problematic to recruit for, and the inclusion of a nontherapeutic intervention as control might have raised ethical issues. In the current study, we found a placebo response of nearly $40 \%$ by midazolam during the first infusion, which is within the range of $35-40 \%$ response rates in antidepressant trials ${ }^{43}$ but greater than previously reported in ketamine studies. For instance, Murrough and colleagues found a $28 \%$ response after a single midazolam infusion compared to $64 \%$ response to ketamine. As placebo response rates increases with the likelihood of receiving active treatment ${ }^{44}$, we can only speculate that the expectation to receive at least one ketamine treatment may have enhanced the active placebo effect of midazolam particularly during the first infusion. Remarkably, despite cumulative active placebo response by midazolam, a single ketamine as the last infusion provide further antidepressant gains and erased any statistical difference in favor of six ketamine up until that point.

The study also allowed concomitant psychiatric medication regimen on stable dosages for at least 6 weeks prior to study onset. While the intention was to recreate realclinical settings with ketamine as an augmenting intervention, we cannot rule out the impact of concurrent medications. For instance, preliminary data reported that benzodiazepines could attenuate the response to ketamine ${ }^{45}$, a finding we did not replicate in this study. Because the study was conducted at a single VA site, results should be carefully extrapolated to other VA sites or non-Veterans. The population studied was predominantly male (85\%) and older (average age of 53-years old), compared to study population in previous trials of TRD and ketamine in which younger participants (i.e., 43years old ${ }^{5}$ ) and the number of women typically exceed the proportion of male participants. Clinical studies have not corroborated sex differences in ketamine response ${ }^{46}$. Another significant difference is that $20 \%$ of patients had sub-syndromal PTSD. While few and small studies have shown the efficacy of single ${ }^{47}$ and repeated ketamine ${ }^{48}$ in PTSD to be comparable with depression, it is unclear whether MDD and comorbid PTSD symptoms could have changed the treatment response to ketamine.

\section{Conclusion}

While acute repeated ketamine showed greater antidepressant efficacy to midazolam after five infusions, this antidepressant difference fell short of significance when a single ketamine was added to the midazolam group as the last infusion. Larger studies would be needed to confirm or not whether repeated ketamine appears to prolong response compared to single ketamine. Increasing knowledge on the mechanism of ketamine should drive future studies on the optimal balance of dosing ketamine for maximum antidepressant efficacy with minimum exposure.

\section{Acknowledgements}

The authors thank the Veterans and their families for participating in this study; nursing staff and clerkship personnel of the $3 \mathrm{E}$ and $3 \mathrm{~K}$ Units at the Minneapolis VA Medical Center whose support made this study possible; Debra L. Condon, MSN, RN, CCRP for assisting with phlebotomy; and study assessors and recruiters for their invaluable contributions to data collection. This work was supported by U.S. Department of Veterans Affairs Clinical Sciences Research and Development Merit Review Award (grant 101 CX001191 to Dr. Shiroma). The funding organizations had no role in the design and conduct of the study; collection, management, analysis and interpretation of the data; preparation, review or approval of the manuscript; and decision to submit the manuscript for publication.

\section{Author details}

${ }^{1}$ Geriatric Psychiatrist, Minneapolis VA Health Care System, Mental Health Service Line, Minneapolis, MN, USA. ${ }^{2}$ Assistant Professor, Department of Psychiatry, University of Minnesota, Minneapolis, MN, USA. 'Statistician/ Research Methodologist, Minneapolis VA Health Care System, Mental Health Service Line; and Assistant Professor/Research Associate, Department of Psychiatry, University of Minnesota, Minneapolis, MN, USA. " Staff

Anesthesiologist, Minneapolis VA Health Care System, Mental Health Service Line; and Clinical Instructor, University of Minnesota Medical School, Minneapolis, MN, USA. ${ }^{5}$ Department of Psychiatry, University of Minnesota, Minneapolis, MN, USA. ${ }^{6}$ Staff Psychologist, Minneapolis VA Health Care System, Mental Health Service Line; and Associate Professor of Psychiatry, University of Minnesota Medical School, Minneapolis, MN, USA. ${ }^{7}$ Senior Research Fellow, Queensland Brain Institute, The University of Queensland, Queensland, Australia; and Assistant Professor Psychiatry, Psychology and Pharmacology Translational Neuroscience Laboratory, Mayo Clinic, Rochester, MN, USA. ${ }^{8}$ Drs. T.J. and Ella M. Arneson Land-Grant Chair in Human Behavior, Professor of Psychiatry, Vice Chair for Research Department of Psychiatry, University of Minnesota, Minneapolis, MN, USA

\section{Data availability}

The authors had full access to the data that were used in the current analysis.

\section{Conflict of interest}

The authors declare that they have no conflict of interest.

\section{Publisher's note}

Springer Nature remains neutral with regard to jurisdictional claims in published maps and institutional affiliations.

Supplementary Information accompanies this paper at (https://doi.org/ 10.1038/s41398-020-00897-0). 
Received: 13 December 2019 Revised: 28 April 2020 Accepted: 7 May 2020 Published online: 26 June 2020

\section{References}

1. Murrough, J. W. et al. Antidepressant efficacy of ketamine in treatmentresistant major depression: a two-site randomized controlled trial. Am. J. Psychiatry https://doi.org/10.1176/appi.ajp.2013.13030392 (2013).

2. Zarate, C. A. Jr. et al. A double-blind, placebo-controlled study of memantine in the treatment of major depression. Am. J. Psychiatry 163, 153-155 (2006).

3. Zarate, C. A. Jr. et al. A randomized trial of an N-methyl-D-aspartate antagonist in treatment-resistant major depression. Arch. Gen. Psychiatry 63, 856-864 (2006).

4. Shiroma, P. R. et al. Augmentation of response and remission to serial intravenous subanesthetic ketamine in treatment resistant depression. J. Affect Disord. 155, 123-129 (2014).

5. Singh, J. B. et al. A Double-blind, randomized, placebo-controlled, dosefrequency study of intravenous ketamine in patients with treatment-resistant depression. Am. J. Psychiatry https://doi.org/10.1176/appi.ajp.2016.16010037 (2016).

6. Phillips, J. L. et al. Single, repeated, and maintenance ketamine infusions for treatment-resistant depression: a randomized controlled trial. Am. J. Psychiatry 176, 401-409 (2019)

7. Zheng, W. et al. Rapid and longer-term antidepressant effects of repeateddose intravenous ketamine for patients with unipolar and bipolar depression. J. Psychiatr. Res. 106, 61-68 (2018).

8. Kanto, J. H. Midazolam: the first water-soluble benzodiazepine. Pharmacology, pharmacokinetics and efficacy in insomnia and anesthesia. Pharmacotherapy 5, 138-155 (1985).

9. Sackeim, H. A. The definition and meaning of treatment-resistant depression. J. Clin. Psychiatry 62(Suppl 16), 10-17 (2001).

10. Rush, A. J., Gullion, C. M., Basco, M. R., Jarrett, R. B. \& Trivedi, M. H. The Inventory of Depressive Symptomatology (IDS): psychometric properties. Psychological Med. 26, 477-486 (1996).

11. Fava, M. et al. Double-blind, placebo-controlled, dose-ranging trial of intravenous ketamine as adjunctive therapy in treatment-resistant depression (TRD). Mol. Psychiatry https://doi.org/10.1038/s41380-018-0256-5 (2018).

12. Aldrete, J. A. The post-anesthesia recovery score revisited. J. Clin. Anesthesia 7, 89-91 (1995).

13. Montgomery, S. A. \& Asberg, M. A new depression scale designed to be sensitive to change. Br. J. Psychiatry. 134, 382-389 (1979).

14. Guy, W. ECDEU Assessment Manual for Psychopharmacology. (US Department of Heath, Education, and Welfare Public Health Service Alcohol, Drug Abuse, and Mental Health Administration, 1976).

15. Jensen, M. P. \& McFarland, C. A. Increasing the reliability and validity of pain intensity measurement in chronic pain patients. Pain 55, 195-203 (1993).

16. Beck, A. T., Epstein, N., Brown, G. \& Steer, R. A. An inventory for measuring clinical anxiety: psychometric properties. J. Consulting Clin. Psychol. 56, 893-897 (1988).

17. Devilly, G. J. \& Borkovec, T. D. Psychometric properties of the credibility/ expectancy questionnaire. J. Behav. Ther. Exp. Psychiatry 31, 73-86 (2000).

18. Collie, A., Maruff, P., Darby, D. G. \& McStephen, M. The effects of practice on the cognitive test performance of neurologically normal individuals assessed at brief test-retest intervals. J. Int. Neuropsychological Soc. 9, 419-428 (2003).

19. Overall, J. E. G. D. R. The Brief Psychiatric Rating Scale. Psychol. Rep. 10, 799-812 (1962).

20. Bremner, J. D. et al. Measurement of dissociative states with the ClinicianAdministered Dissociative States Scale (CADSS). J. Trauma. Stress 11, 125-136 (1998).

21. Young, R. C., Biggs, J. T., Ziegler, V. E. \& Meyer, D. A. A rating scale for mania: reliability, validity and sensitivity. Br. J. Psychiatry 133, 429-435 (1978).

22. Kelly Posner et al. The Columbia-Suicide Severity Rating Scale: initial validity and internal consistency findings from three multisite studies with adolescents and adults. Am. J. Psychiatry 168, 1266-1277 (2011).

23. Morgan, C. J., Muetzelfeldt, L. \& Curran, H. V. Consequences of chronic ketamine self-administration upon neurocognitive function and psychological wellbeing: a 1-year longitudinal study. Addiction 105, 121-133 (2010).

24. Li, J. H. et al. To use or not to use: an update on licit and illicit ketamine use. Subst. Abus. Rehabilit. 2, 11-20 (2011).
25. Zhang, M. W., Harris, K. M. \& Ho, R. C. Is off-label repeat prescription of ketamine as a rapid antidepressant safe? Controversies, ethical concerns, and legal implications. BMC Med. Ethics 17, 4 (2016).

26. Diagnostic and Statistical Manual of Mental Disorders, Fourth Edition, Text Revision (DSM-IV-TR). (American Psychiatric Association, 2000).

27. Rasmussen, K. G. et al. Serial infusions of low-dose ketamine for major depression. J. Psychopharmacology https://doi.org/10.1177/0269881113478283 (2013).

28. Murrough, J. W. et al. Rapid and longer-term antidepressant effects of repeated ketamine infusions in treatment-resistant major depression. Biol. Psychiatry 74, 250-256 (2013).

29. Huitfeldt, B. \& Montgomery, S. A. Comparison between zimeldine and amitriptyline of efficacy and adverse symptoms-a combined analysis of four British clinical trials in depression. Acta Psychiatr. Scand. Suppl. 308, 55-69 (1983).

30. Duru, G. \& Fantino, B. The clinical relevance of changes in the MontgomeryAsberg Depression Rating Scale using the minimum clinically important difference approach. Curr. Med. Res. Opin. 24, 1329-1335 (2008).

31. Vanina Popova, M. D. et al. Efficacy and safety of flexibly dosed esketamine nasal spray combined with a newly initiated oral antidepressant in treatmentresistant depression: a randomized double-blind active-controlled study. Am. J. Psychiatry 176, 428-438 (2019).

32. Wilkinson, S. T. et al. Impact of midazolam vs. saline on effect size estimates in controlled trials of ketamine as a rapid-acting antidepressant. Neuropsychopharmacology 44, 1233-1238 (2019).

33. Su, T. P. et al. Dose-related effects of adjunctive ketamine in taiwanese patients with treatment-resistant depression. Neuropsychopharmacology $\mathbf{4 2}$, 2482-2492 (2017).

34. Luckenbaugh, D. A. et al. Do the dissociative side effects of ketamine mediate its antidepressant effects? J. Affect. Disord. 159, 56-61 (2014).

35. Niciu, M. J. et al. Features of dissociation differentially predict antidepressant response to ketamine in treatment-resistant depression. J. Affect. Disord. 232, 310-315 (2018).

36. Daly, E. J. et al. Efficacy of esketamine nasal spray plus oral antidepressant treatment for relapse prevention in patients with treatment-resistant depression: a randomized clinical trial. JAMA Psychiatry https://doi.org/10.1001/ jamapsychiatry.2019.1189 (2019).

37. Li, N. et al. Glutamate N-methyl-D-aspartate receptor antagonists rapidly reverse behavioral and synaptic deficits caused by chronic stress exposure. Biol. Psychiatry 69, 754-761 (2011).

38. Duman, R. S., Li, N., Liu, R. J., Duric, V. \& Aghajanian, G. Signaling pathways underlying the rapid antidepressant actions of ketamine. Neuropharmacology 62, 35-41 (2012).

39. Moda-Sava, R. N. et al. Sustained rescue of prefrontal circuit dysfunction by antidepressant-induced spine formation. Science https://doi.org/10.1126/ science.aat8078 (2019).

40. Williams, N. R. et al. Attenuation of antidepressant effects of ketamine by opioid receptor antagonism. Am. J. Psychiatry 175, 1205-1215 (2018).

41. Yoon, G., Petrakis, I. L. \& Krystal, J. H. Association of combined naltrexone and ketamine with depressive symptoms in a case series of patients with depression and alcohol use disorder. JAMA Psychiatry 76, 337-338 (2019).

42. Zanos, P. et al. NMDAR inhibition-independent antidepressant actions of ketamine metabolites. Nature 533, 481-486 (2016).

43. Furukawa, T. A. et al. Placebo response rates in antidepressant trials: a systematic review of published and unpublished double-blind randomised controlled studies. Lancet Psychiatry 3, 1059-1066 (2016).

44. Papakostas, G. I. \& Fava, M. Does the probability of receiving placebo influence clinical trial outcome? A meta-regression of double-blind, randomized clinical trials in MDD. Eur. Neuropsychopharmacol. 19, 34-40 (2009).

45. Albott, C. S. et al. The antidepressant effect of repeat dose intravenous ketamine is delayed by concurrent benzodiazepine use. J. Clin. Psychiatry 78, e308-e309 (2017)

46. Niciu, M. J. et al. Clinical predictors of ketamine response in treatment-resistant major depression. J. Clin. Psychiatry 75, e417-e423 (2014).

47. Feder, A. et al. Efficacy of intravenous ketamine for treatment of chronic posttraumatic stress disorder: a randomized clinical trial. JAMA Psychiatry 71, 681-688 (2014).

48. Albott, C. S. et al. Efficacy, safety, and durability of repeated ketamine infusions for comorbid posttraumatic stress disorder and treatment-resistant depression. J. Clinical Psychiatry https://doi.org/10.4088/JCP.17m11634 (2018). 